\title{
Predicción de la lealtad de los usuarios de centros deportivos privados. Variables de gestión y bienestar subjetivo \\ Prediction of the loyalty of costumers of private sports centers. Management variables and subjective well-being \\ *Fernando García Pascual, **Mario Alguacil, *** Núria Molina García \\ *Universidad de Valencia (España), **Universidad Católica de Valencia (España), ***Universidad de Alicante (España)
}

Resumen. Una de las finalidades más importantes en el contexto de los servicios deportivos es conocer cuáles son las intenciones futuras del usuario, por ello tratar de analizar la lealtad del usuario, se ha convertido en una de los objetivos principales dentro de la gestión del deporte, en concreto, los centros deportivos. Por ello, el objetivo es conocer qué influencia tienen las demás variables de gestión y el bienestar subjetivo sobre esta dimensión que mide las intenciones futuras del usuario, como es la lealtad. La muestra de este trabajo estaba formada por 303 usuarios de la instalación deportiva (164 hombres, 139 mujeres). Para el análisis estadístico se utilizó con el programa SPSS v.23. Todas las variables que formaban el modelo obtuvieron buenos resultados en cuanto a la fiabilidad, siendo el valor emocional el que mayor valor de alfa de Cronbach obtuvo. Tras calcular los estadísticos descriptivos de las diferentes escalas del modelo, se realiza un análisis de regresión múltiple, y se observa como este explica el 38\% de la varianza, mostrando todas las variables significatividad con la lealtad del usuario, menos la variable que mide el valor precio. Tras conocer que variables influyen más en la predicción de la lealtad, ofrecer a los gestores deportivos información acerca de qué variables reforzar, ayudará a obtener usuarios más leales y en consecuencia fieles con el servicio.

Palabras clave. Lealtad, centros deportivos, gestión del deporte, valor emocional, satisfacción.

\begin{abstract}
One of the most important purpose in the context of sports services is to know what the costumer's future intentions are, so trying to analyze costumer loyalty has become one of the main objectives within the management of sport, specifically sports centers. Therefore, the aim is to know what influence the other management variables and subjective well-being have on this dimension that measures the future intentions of the costumer, such as loyalty. The sample of this work was made up of 303 costumers of the sports centre (164 men, 139 women). For statistical analysis it was used with SPSS v.23. All the variables that formed the model obtained good results in terms of reliability, being the emotional value the one that obtained the highest alpha value of Cronbach. After calculating the descriptive statistics of the different scales of the model, a multiple regression analysis is carried out, and it is observed how this one explains $38 \%$ of the variance, showing all the variables significance with the loyalty of the user, excepting the variable that measures the price value. After knowing which variables influence loyalty prediction, providing sports managers with information about which variables to reinforce will help to obtain more loyal costumers and consequently faithful to the service.
\end{abstract}

Key words. Loyalty, sports centres, sport management, emotional value, satisfaction.

\section{Introducción}

Actualmente, existen multitud de servicios deportivos que son ofrecidos a la sociedad con distintas finalidades. Todas estas organizaciones deportivas trabajan con clientes/usuarios, ofreciéndoles una contraprestación por un servicio abonado, por ello, una de las principales finalidades de estos servicios deportivos, es obtener un público satisfecho y en consecuencia fiel al servicio. Ya que como indica Juliá y Porsche (2002), las empresas se preocupan de dicha fidelidad, ya que es más costoso tener nuevos clientes que mantenerlos. En la literatura existente de la gestión del deporte, hay cada vez más estudios relacionados en el análisis de la lealtad de los usuarios, ya que ofrece resultados subjetivos interesantes acerca de cuáles son sus intenciones futuras con el servicio. Este trabajo tiene como objeto de estudio los centros deportivos, instalaciones que desde hace un tiempo se han convertido en auténticos templos del deporte, por su gran variedad de oferta deportiva adaptada a todos los perfiles encontrados en la sociedad. España, por lo que refiere a ingresos, se ha situado en la quinta posición europea en el sector del fitness, obteniendo cada año porcentajes mayores de ingresos (Deloitte, 2019), de ahí la importancia de estas instalaciones deportivas.

Fecha recepción: 10-07-19. Fecha de aceptación: 30-10-19
Conocer las percepciones sobre las intenciones que tienen los usuarios de un servicio deportivo, acerca de su continuidad en este, es una de las variables más utilizadas, así como una de las más importantes para el devenir del servicio deportivo. Una de las variables que miden las intenciones futuras de los consumidores es la lealtad. Esta, ha sido una variable muy analizada en distintos trabajos, así mismo, es un concepto que tiene mucha trascendencia en el contexto del marketing (Kumar \& Shah, 2004; Luque \& Castañeda, 2005).

Dentro de la literatura existente acerca de la gestión del deporte, se encuentran trabajos (Avourdiadou \& Theodorakis, 2014; Calabuig, Burillo, Crespo, Mundina, \& Gallardo, 2010; García-Fernández, Gálvez-Ruiz, Vélez-Colón, Ortega-Guitérrez, Fernández-Gavira, 2018) que analizan la lealtad desde el comportamiento, defendiendo que esta variable comprende repetidas compras o reiteración de esas intenciones. Así también, García, Fernández y Bernal, (2014), argumentan que en cuanto al género las mujeres tienen mejores intenciones futuras en el contexto de los centros fitness. García-Pascual, Molina y Mundina (2019) también analizan en su trabajo realizado en centros deportivos, observando las percepciones de los usuarios, como la satisfacción y el valor percibido de los mismos, son las variables que más influyen en las intenciones futuras de estos usuarios hacia el servicio deportivo.

La satisfacción, según la literatura en gestión del deporte, es una variable predictora de las intenciones futuras de 
los clientes (Calabuig, Molina y Nuñez, 2012; GarcíaFernández et al., 2018), por ello y, en consecuencia, cuanto más satisfechos estén los clientes más posibilidad de garantizar las intenciones de volver a adquirir/repetir dicho servicio.

Tsiros y Mittal (2000) concluyen que en función del nivel de satisfacción del consumidor puede influir en comportamientos futuros, tanto positivos como la repetición de compra o más influyentes como la conducta de queja. Calabuig, Quintanilla y Mundina (2008) defienden que, desde hace ya un tiempo, la satisfacción, es una de las variables más estudiadas dentro del contexto de las organizaciones deportivas, ya que ofrece un resultado en el que el usuario, de manera subjetiva mide su experiencia con dicha organización. De manera más específica, dentro del contexto de los servicios deportivos, en concreto, los centros deportivos, también han sido multitud los autores que han analizado dichas variables de gestión (Silla et al.,2014; Leyton, García, Fuentes y Jiménez, 2018; Haro, Pérez, Leyton, Caamaño y Nuviala, 2018).

El valor que percibe y otorga el usuario al servicio que ha consumido también guarda una relación estrecha con las intenciones de estos en volver a adquirir el servicio. Siendo así también, este valor percibido del usuario, un antecedente directo de las intenciones futuras de estos usuarios de seguir en el servicio deportivo. Existen múltiples trabajos que evidencian de manera positiva el vínculo del valor percibido del servicio con las intenciones futuras de los usuarios (Calabuig, Núñez-Pomar, Prado-Gascó \& Añó 2014; Yu, Zhang, Kim, Chen, Henderson, Min \& Huang, 2014)

Por otro lado, se observan muchos trabajos, donde tratan de analizar aspectos comportamentales, así como analizar las percepciones acerca de un servicio, pero cada vez es más habitual observar dentro de trabajos acerca de los servicios deportivos, el análisis de ciertas variables psicológicas.

Pese a su escasez de estudio dentro de la literatura existente, durante los últimos años, ha crecido de manera muy progresiva, el interés de muchos autores para medir el bienestar subjetivo de los clientes, ya que ofrecen valoraciones más amplias incluso fuera del contexto analizado.

Lyubomirsky, King y Diener (2005), confirman que el bienestar subjetivo no solo tiene efecto durante el proceso de satisfacción en la vida, sino que también tiene sus consecuencias para alcanzar resultados positivos. Por ello, las personas felices son aquellas en las que predominan en su vida aspectos placenteros y positivos, sobre los negativos. A diferencia de otras disciplinas, esta variable de bienestar subjetivo ha sido muy poco analizada en la literatura de la gestión del deporte, pero desde hace unos años diferentes trabajos tratan de analizar la relación del bienestar subjetivo en los servicios deportivos (Molina, 2016; Hamer y Stamatakis, 2010, García-Pascual, Silla, Mundina y Escamilla, 2016). Concluyendo, algunos de estos trabajos, que los estados emocionales, así como los resultados obtenidos en dichos servicios deportivos, son las variables que más influyen para tener alto niveles de bienestar personal por parte del usuario de la instalación deportiva.

Por ello, este trabajo tiene como objetivo, analizar dentro del ámbito de los centros deportivos, cómo influyen las diferentes variables de gestión y el bienestar subjetivo en la lealtad de los usuarios hacia el servicio deportivo. Para así aportar a la literatura existente, resultados que ayuden a estas organizaciones a poder aumentar la fidelidad de los usuarios hacia estos servicios deportivos.

\section{Material y método}

\section{Participantes}

La muestra de la investigación es de 303 usuarios de un centro deportivo, de gestión privada, ubicado en una localidad cercana al área metropolitana de Valencia. Con una edad media de 35,89 años $( \pm 15,79)$, de los cuales 164 son hombres (54\%) y 139 son mujeres (46\%). En cuanto a la práctica deportiva semanal, el $84 \%$ de los encuestados afirmaron que acudían 3 veces o más a la semana a realizar actividad física a la instalación deportiva. Siendo los usuarios que han obtenido estudios universitarios los que han tenido mayor participación en la encuesta (41\%).

\section{Instrumento}

El cuestionario estaba formado por 17 indicadores, tomados de diferentes escalas ya validadas y analizadas en otros trabajos. En dicho instrumento se utilizaron las escalas para medir la satisfacción general, formado por 2 ítems (Hightower, Brady y Baker, 2002), el valor percibido (Sweeney y Soutar, 2001) que analiza el valor percibido del consumidor a través de sub-dimensiones (7 ítems) como el valor emocional, valor precio y valor social. Las intenciones futuras, formada por 4 ítems (Zeithaml, Berry y Parasuraman, 1996), y el bienestar subjetivo (Personal Wellbeing Index) de cada uno de los usuarios/as, con 8 ítems (Cummins, 2006). Todas las respuestas eran tipo Likert, siendo el valor mínimo 1 (Totalmente desacuerdo) y el valor máximo 5 (Totalmente de acuerdo), salvo en la escala que mide el bienestar subjetivo cuyo valor mínimo es 1, y el valor máximo, 10.

\section{Procedimiento}

Para la recopilación de datos, se contactó con la dirección del centro deportivo, y tras su aceptación, se suministró los instrumentos a los usuarios en la misma instalación, en diferentes franjas horarias, para abarcar todos los perfiles que acuden a la instalación deportiva. Se utilizó un muestreo de conveniencia no probabilístico, ya que se pedía la participación a aquellos usuarios que entraban o salían de la propia instalación para pedir sus percepciones acerca de la misma, ya que el centro disponía de un elevado número de usuarios inscritos. Durante este proceso, los investigadores estuvieron en todo momento pendiente de los usuarios, ayudándoles en caso necesario. Así también, se les garantizó máxima confidencialidad de los datos.

\section{Análisis de datos}

Tras conocer las percepciones de los usuarios del centro deportivo, se procedió a analizar los diferentes resultados obtenidos, para ello primero se obtuvieron los descriptivos a través del programa SPSS (Statistical Package for the Social Sciences) v.23. En primer lugar, se comprueba la consistencia interna de las diferentes escalas que componen el instrumento de medida, para así poder conocer la fiabilidad y la validez de las escalas que componen el instrumento de medida. A continuación, se realizó un análisis de correlación, 
mostrando la correlación de Pearson. Por último, también se utilizó el programa SPSS para realizar la regresión lineal múltiple, cuya finalidad es definir un modelo que argumente el comportamiento de la variable dependiente (lealtad), en función de la información que facilitan las variables independientes (satisfacción, valor percibido y bienestar subjetivo).

\section{Resultados}

Como se observa en la tabla 1 , las dimensiones que conforman esta escala obtuvieron valores de alfa favorables, la dimensión que mide el valor emocional obtuvo $\alpha=, 89$ para el valor precio resultó un $\alpha=, 78$ en cuanto al valor social se obtuvo $\alpha=$, 78, el bienestar subjetivo representó $\alpha=$, 72, la lealtad obtuvo un $\alpha=$, 86 y por último la satisfacción del usuario resultó $\alpha=$, 80. Por tanto se confirma que existe buena consistencia interna ya que los valores de Alfa de Cronbach, son superiores a ,70 punto de corte sugerido por Hair, Black, Babin, Anderson y Tatham (2006).

En cuanto a la fiabilidad compuesta (FC) como bien se representa en la tabla 3 , se observa como los valores están comprendidos por encima de ,70 punto de corte sugerido por la literatura (Hair et al., 2006). Así también, observando la varianza media extraída (AVE) de las dimensiones, encontramos que las escalas tienen valores superiores a .50, punto de corte indicado por Fornell y Larcker (1981).

Previo al análisis de la regresión lineal múltiple, se realizó una correlación de Pearson entre las escalas utilizadas. Como se observa en la tabla 2, todas las variables muestran una relación estadísticamente significativa, siendo dos de las dimensiones del valor percibido (emocional y social) junto con la satisfacción del usuario las que mayor correlación muestran con la lealtad. Por otro lado, el valor precio y el bienestar subjetivo son las variables que menor correlación ha obtenido con la variable que mide las intenciones futuras.

Una vez analizada la fiabilidad de las escalas utilizadas y mostrada su correlación, se procede a realizar una regresión lineal múltiple, como se observa en la tabla 3. Este modelo que analiza la relación de las dimensiones de la satisfacción general, el valor percibido y el bienestar subjetivo con la lealtad. Se encuentra que es un modelo significativo $(F(5)=$

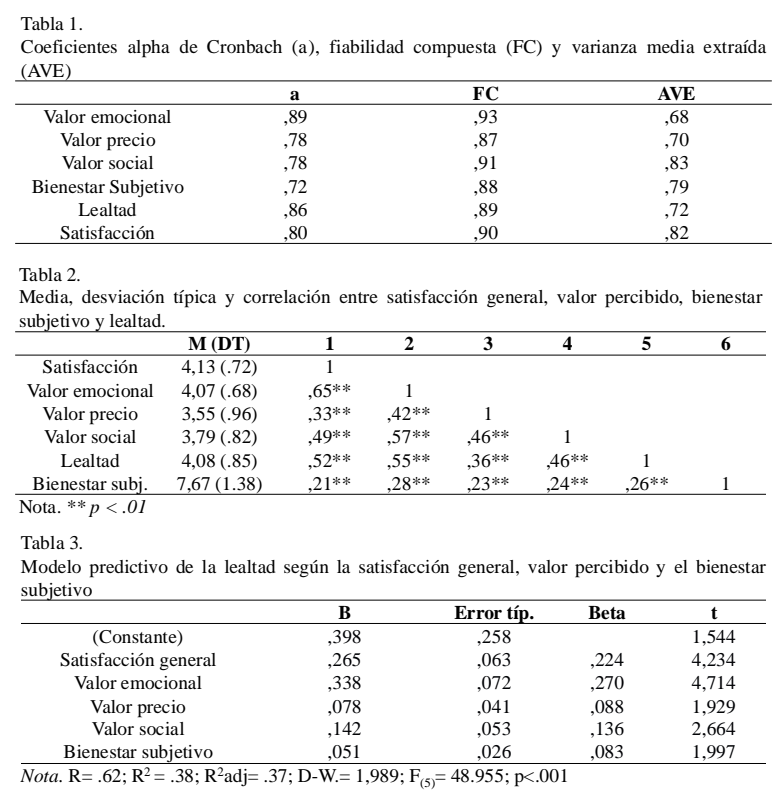

1,989; $p<.001$ ) y explica el 38\% de la varianza. Siendo las variables independientes de Satisfacción general, Valor emocional, Valor social y Bienestar subjetivo las que han mostrado significatividad en relación con la lealtad de los usuarios de la instalación deportiva, no mostrando ninguna relación significativa con la dimensión de Valor precio. Dentro de las variables que han mostrado significatividad encontramos que la que mayor peso predictivo ofrece es el Valor emocional $(\beta=.27)$, seguida de la Satisfacción general $(\beta=.22)$, el Valor social $(\beta=.14)$ y por último el Bienestar subjetivo $(\beta=$ $.08)$.

\section{Discusión}

Revisando la literatura, y observando los comportamientos de los distintos servicios deportivos actuales, se observa como la fidelidad es una variable muy valorada y analizada en dicho sector. Cada vez son más los servicios deportivos que tratan de obtener una mayor retención de clientes y conseguir un cliente fiel al servicio para un largo periodo de tiempo. Pero para conseguir dicha lealtad, existen diferentes variables que ayudan a que se produzca esa fidelidad del cliente con el servicio ofrecido. En este trabajo se observa como el valor emocional, la satisfacción del usuario, el valor social y el bienestar subjetivo predicen significativamente la lealtad del usuario hacia el servicio deportivo ofrecido, es decir, cuanto más se trabaje sobre estas variables, más positivas serán las intenciones futuras del usuario con el servicio.

Los resultados obtenidos en este trabajo corroboran conclusiones que anteriormente se han obtenido en otros trabajos encontrados en la literatura existente en la gestión del deporte, como que el valor percibido tiene relación directa y predice significativamente las intenciones futuras (Calabuig et al., 2015; Yacout, 2010) así como también la satisfacción del usuario (Calabuig et al., 2012; García-Fernández et al., 2018).

En este trabajo, en referencia a todas las variables, se ha observado como la dimensión del valor percibido del usuario, el valor emocional, es la que predice con mayor peso las intenciones futuras de los usuarios. El factor emocional, es una variable con gran influencia sobre la lealtad de los usuarios hacía el servicio deportivo, dicha dimensión de valor analiza el bienestar, el placer, la felicidad que le aporta acudir a la instalación deportiva. Esta dimensión del valor percibido del servicio tiene una gran significatividad con la lealtad del usuario del centro deportivo por los servicios que recibe. Este resultado coincide con los obtenidos en los trabajos de otros autores que confirman la relación directa del valor percibido con las intenciones futuras (Molina, Mundina, GarcíaPascual \& Alejos, 2016; Yacout, 2010). En este trabajo, se encuentra como el valor precio no predice significativamente la lealtad, aunque los valores obtenidos no distan mucho de predecirla, este hecho puede ocurrir, por el amplio abanico de precios que oferta la instalación deportiva, no observando los usuarios en ese aspecto económico, un elemento clave para ser fiel al servicio.

Es interesante observar como el valor percibido está situándose como una variable clave para la predicción de las intenciones futuras de los usuarios de servicios deportivos, incluso con mayor peso que la satisfacción. Aunque la litera- 
tura justifica mediante multitud de trabajos, que el valor es un claro antecedente de la satisfacción del usuario (GarcíaFernández, Gálvez-Ruiz, Fernández-Gavira, Vélez-Colón, Pitts \& Bernal-García, 2018; García-Pascual et al., 2019).

En el presente trabajo, la satisfacción del usuario también predice de manera significativa a la lealtad del usuario, por ello se confirma los resultados de otros estudios existentes en la literatura en el que la satisfacción tiene una relación directa y positiva sobre las intenciones futuras de los usuarios (Theodorakis, Howat, Ko \& Avourdiadou, 2014; Yoshida, 2017). Estos datos confirman que cuanto más satisfechos estén los clientes más influirán en las intenciones futuras de los mismos, siendo más leales al servicio (Tsiros \& Mittal, 2000). Por lo tanto, recalcar la importancia de la variable intenciones futuras de los usuarios de las instalaciones deportivas y todas aquellas que la predicen, ya que como afirmó Calabuig et al. (2015) cuando se obtienen unos elevados valores de esta variable, se garantiza el funcionamiento y la viabilidad de dichas instalaciones deportivas.

Por lo que refiere al bienestar subjetivo, observamos que la escala que lo medía ha obtenido buenas propiedades psicométricas, con una correcta consistencia interna. Esta escala fue elaborada para poder medir la felicidad en ciertos aspectos de su vida como la seguridad, el nivel de vida, las creencias religiosas, la salud, entre otros. En cuanto a la predicción de la lealtad, se observa como dicha variable sí que la predice significativamente, confirmando así, los resultados obtenidos en otros trabajos que se encuentran en la literatura (White \& Yu, 2019). Dichos resultados reafirman que tener usuarios dentro del servicio con niveles positivos en cuanto a su satisfacción con la vida y felices, ayudará a que estos usuarios sean fieles al servicio, y por tanto, permanezcan en el mismo.

\section{Conclusión}

Como conclusión, recalcar que la lealtad es una variable muy influyente en la gestión del deporte y en la retención de clientes por parte de un servicio. Destacar la importancia de los indicadores que miden el valor emocional en cuanto a la predicción de la lealtad, siendo la satisfacción de los usuarios, la siguiente variable más importante en esta predicción. Por ello, este trabajo, trata de transmitir a los gestores de estos servicios, los centros deportivos, la importancia de enfatizar aspectos psicológicos como el placer, la felicidad, el bienestar propio del cliente, a través de los diferentes programas y actividades que se desarrollan en estas instalaciones deportivas.Sin restar importancia, a mantener un usuario satisfecho con el servicio, a través de su experiencia con el centro deportivo, ya que es un gran indicador de la calidad del servicio obtenido. Facilitando así, que el usuario sea fiel al servicio y en consecuencia tenga actitud de repetición de compra con respecto al mismo, y poder prolongar durante el mayor tiempo posible su pertenencia. Se ha observado como este trabajo ofrece resultados significativos para la literatura existente en gestión del deporte, pero existen ciertas limitaciones en el mismo. Este estudio se ha realizado en un centro deportivo de la provincia de Valencia, así como su gestión era de carácter privada, por ello en futuras líneas de investigación, debería orientarse a poder encuestar a los usuarios de diferentes centros deportivos a nivel nacional, y poder encuestar a usuarios tanto de instalaciones deportivas con gestión privada y pública y así poder diferenciar resultados y observar si las percepciones de ambos distan unas de otras.

\section{Referencias}

Avourdiadou, S. \& Theodorakis, N. D. (2014). The development of loyalty among novice and experienced customers of sport and fitness centers. Sport Management Review, 17(4), 419-431.

Calabuig, F., Burillo, P., Crespo, J., Mundina, J. J., \& Gallardo, L. (2010). Satisfacción, calidad y valor percibido en espectadores de atletismo. Revista Internacional de Medicina y Ciencias de la Actividad Física y el Deporte, 10(40), 577-593.

Calabuig, F., Molina, N. \& Núñez, J. (2012). Una aplicación inicial del modelo tridimensional de calidad de servicio en centros deportivos privados. E-balonmano.com: Journal of Sport Science, 8 (1), 67-8

Calabuig, F., Prado-Gascó, V., Crespo, J., Núñez-Pomar, J. \& Añó, V. (2015). Spectator emotions: Effects on quality, satisfaction, value and future intentions. Journal of Business Research, 68(7), 1445-1449

Deloitte. (2019). EuropeActive European Health \& Fitness Market Report 2019.

Fornell, C. \& Larcker, D. F. (1981). Evaluating structural equation models with unobservable variables and measurement error. Journal of marketing research, 39-50.

García, J., Fernández, J. \& Bernal, A. (2014). La percepción de calidad y fidelidad en clientes de centros de fitness low-cost. Suma Psicológica, 21(2), $123-130$.

García-Fernández, J., Gálvez-Ruiz, P., Vélez-Colón, L., Ortega-Fernández, J. \& Fernández-Gavira, J. (2018). Exploring fitness centre consumer loyalty: diferences of non-proit and low-cost business models in Spain. Economic Research, 31 (1), 1042-1058.

García-Fernández, J., Gálvez-Ruíz, P., Fernández-Gavira, J.,Vélez Colón, L., Pitts, B., \& Bernal-García, A. (2018). The effects of service convenience and perceived quality on perceived value, satisfaction and loyalty in lowcost fitness centers. Sport Management Review, 21(3),250-262.

García-Pascual, F., Silla, A., Mundina, J. \& Escamilla, P. (2016). El efecto de variables de gestión en el bienestar subjetivo de usuarios de centros deportivos. Journal of Sports Economics \& Managament, 6 (2), 99-110.

García-Pascual, F., Molina, N. \& Mundina, J. (2019). Influencia de la satisfacción y el valor percibido sobre el «Word of Mouth» en los usuarios de centros deportivos. SPORT TK- Revista euroamericana de ciencias del deporte, 8 (1), 29-36.

Hair, J. F., Black, W. C., Babin, B. J., Anderson, R.E. \& Tatham, R. L. (2006). Multivariate data analysis (6 $6^{a}$ Edición). Upper Saddle River, NJ: Pearson-Prentice-Hall.

Haro, G. M., Pérez. O. R., Leyton, R. M., Caamaño, G. A., \& Nuviala, A. (2018). Grupos de usuarias del mercado deportivo femenino. Retos, (34), 123-127.

Kumar, V. \& Shah, D. (2004). Building and sustaining profitable customer loyalty for the 21st century. Journal of Retailing, 80(4), 317-330.

Leyton, R. M., García, M. J., Fuentes, G. J. P., \& Jiménez, C. R. (2018). Análisis de variables motivacionales y de estilos de vida saludables en practicantes de ejercicio físico en centros deportivos en función del género. Retos, 34 (2), 166-171.

Luque, T. \& Castaneda, J. A. (2005). Análisis del constructo fidelidad conativa en el contexto electrónico. XVII Encuentro de Profesores Universitarios de Marketing,Madrid. Ed. ESIC, Madrid, 793-814.

Lyubomirsky, S. \& Lepper, H. (1999). A measure of subjective happiness: Preliminary reliability and construct validation. Social Indicators Research, 46, 137-155.

Sweeney, J.C. \& Soutar, G. (2001). Consumer perceived value: the development of a multiple item scale, Journal of Retailing, 77, 203-220.

Theodorakis, N. D., Howat, G., Ko, Y. J. \& Avourdiadou, S.(2014). A comparison of service evaluation models in the context of sport and fitness centres in Greece. Managing Leisure, 19(1), 18-35

Tsiros, M. \& Mittal, V. (2000). Regret: a model of its antecedents and consequences in consumer decision making, Journal of Consumer Research, 26(4), 401-417.

Yacout, O. (2010). Service quality, relational benefits, and customer loyalty in a non-western context. Society for the Advancement of Management, 75, 4-22.

Yoshida, M. (2017). Consumer experience quality: A review and extension of the sport management literature. Sport Management Review, 20(5), 427442.

Yu, H. S., Zhang, J. J., Kim, D. H., Chen, K. K., Henderson, C., Min, S. D. \& Huang, H. (2014). Service quality, perceived value, customer satisfaction, and behavioral intention among fitness center members aged 60 years and over. Social Behavior and Personality: an International Journal, 42 (5), 757-767.

Zeithaml, V. A., Bitner, M. J. \& Gremler, D.D (2006). Services Marketing: Integrating Customer Focus across the Firm. 4th edition. New York: McGraw-Hill. 